\title{
MODELLING TECHNICAL COUNTERMEASURES OF ADVANCED PERSISTENT THREATS
}

\author{
Mathew Nicho ${ }^{1}$ and Christopher McDermott ${ }^{2}$ \\ ${ }^{I}$ Zayed University, Dubai, United Arab Emirates \\ ${ }^{2}$ Robert Gordon University, Aberdeen, United Kingdom
}

\begin{abstract}
An advanced persistent threat (APT) is a highly targeted and sophisticated attack directed at the internetworked computer user at the workplace. They typically employ zero-day malware, stealth, and multiple advanced techniques to gain entry and maintain presence undetected inside the network. As a result, preventing their ingress at the network perimeter and detection once infiltration occurs, continues to be a challenge. In this respect, the major objective of this research is to propose a classification schema for the APT technical countermeasures. We interviewed senior managers working in government and private organizations in the United Arab Emirates over a period of four years (2014 to 2017) to gain their perspective of the threat and elicit technical countermeasures. We anticipate the proposed methods and practices can assist organizations, not only in the UAE but also in the wider global context, to implement an appropriate mix of countermeasures for APT threats from a multi-dimensional perspective.
\end{abstract}

\section{KEYWORDS}

Advanced Persistent Threats, Spear Phishing

\section{INTRODUCTION}

Advanced persistent threats are commonly characterized by their use of zero-day malware, multiple threat agents, stealth and persistent nature. Their stealthy, targeted and data focused nature (Chandra et al., 2016), makes detection a challenge using traditional defense methodologies (Zhang et al., 2017). Following infiltration, they endeavor to remain inside the network or repeat intrusion multiple times until they can accomplish their goals (Matsuda et al., 2018). As a highly sophisticated, well-resourced threats aimed primarily towards the government sector (Thakar and Parekh, 2016), the goal of an APT attack is not to just gather a target entity's data, but to accomplish it undetected (Alshamrani et al., 2019). While the majority of cyber-attacks rely on automated scanning and exploitation of known vulnerabilities over large sets of targets (Marchetti et al., 2016), APT attacks are highly targeted attacks, with a clear goal typically targeting governments or business targets with substantial intellectual property value (Chen et al., 2014, Vert et al., 2014). Reports of APT attacks targeting high-profile organizations, ranging from large-scale enterprises and financial institutions to government sectors continue to occur unabated (Yang et al., 2018). While APTs have drawn increased attention from the industrial security community, a comprehensive and clear understanding of the APT research problem is still lacking (Chen et al., 2014). Since they often launch attacks in multiple domains of the target systems and networks, in multiple stages, using packets that may not be malicious, it is extremely challenging for most current IDS to detect them (Aparicio-Navarro et al., 2018).

\section{RESEARCH ON APT COUNTERMEASURES}

An APT attack process progresses through multiple phases (reconnaissance, delivery, exploitation, operation, data collection, and exfiltration) targeting the data (Giura and Wang, 2013). However, it can also traverse any one or more of the planes/dimensions namely the physical plane $(\mathrm{P})$ comprising of servers/hardware, user plane (U) that includes users and user systems, network plane (N) incorporating LAN/WAN, and application plane (A) which includes software applications, or even any new and emerging planes (Giura and Wang, 2012). 
Technical countermeasures have been proposed by (Huang and Zhu, 2019), where a multi-stage Bayesian game framework can capture incomplete information of deceptive APTs and their multistage multi-phase movement. (Siddiqui et al., 2016) proposed classification of APT based on anomalous traffic patterns using a feature vector obtained during processing of TCP/IP session information. A disguised executable file detection (DeFD) module system, which aims to detect disguised executable files transferred over network connections, has been proposed by (Ghafir et al., 2018). A malicious SSL certificate Detection module, which aims at detecting APT command and control (C\&C) communications, based on a blacklist of malicious SSL certificates has been proposed by (Ghafir et al., 2017). It has been recognized that deception tools like honeypots, can significantly increase the possibility of early detection of such sophisticated attacks. Saud and Islam, (2015) proposed combining honeypots and network intrusion detection systems to alert the administrator by correlating different network events. Matsuda et al., (2018) proposed a new method based on outlier detection and machine learning for detecting attacks that utilize legitimate accounts. A Multi-Layer Defense System which attempts to prevent APTs by analyzing information of network, servers, end-users, and logs, has been proposed by (Moon et al., 2014). Meanwhile, a random forest algorithm has been proposed as a method to detect the presence of APT in infected machines (Chandran et al., 2015). An intrusion detection system has also been designed to minimize the damage from the APT attack by detecting the possibility of initial intrusions (Moon et al., 2015). Security log analysis using Big data has been proposed by Jeon et al. (2016). Here they employed a finite angular state transition velocity machine, and its vector based analytic algebra, to detect APT inside the network (Vert et al., 2014). Network traffic and client data analysis, form multiple network locations, using both signature and anomaly detection methods derived from the intelligent data analysis field, has been proposed by De Vries et al. (2012). An intrusion detection system with the ability to detect the possibility of initial intrusions, as well as using security intelligence and big data analytics was proposed by (Marchetti et al., 2016). An automatic training system that generates simulated training emails using an email client has been proposed by (Iwata et al., 2017). While these security measures and models enhance APT security, we view the problem from the four planes to come up with a classification schema.

\section{FINDINGS AND DISCUSSION}

Nine respondents in the IS security domain from nine diverse organizations, across multiple sectors of industry (financial, media, information technology services, government, aviation, and oil sector) were selected for interview. The interviews were transcribed, validated through subsequent telephonic interviews, and imported to NVIVO 10, a qualitative analysis software used by qualitative researchers. The analysis follows the guidelines of Whittaker (2006), where (1) the data (especially the interview data) was coded, (2) the transcribed text was systematically examined to identify key concepts, (3) the data was grouped into pre-defined constructs and (4) searched for relationships between a category and its concepts to isolate it to its respective planes.

Respondents were unanimous in their assertion that 'zero day' attacks, inherent in many APT threats, cannot be completely prevented (responses from respondents have been inserted using quotation marks). APTs were identified by respondents as being "an advanced form of historical threat, that have been continuing". In this regard, respondents highlighted the uniqueness of APT attacks as "totally different stuff" due to their elusiveness from using zero-day malware, stating they were "quite difficult to prevent" since infection occurs through users. Moreover, they state "while it can't be fully eliminated, there could be potential threats and further penetrations once it enters the system". Regarding commercial APT detection and prevention solutions, respondents stated that "vendors may have APT solutions for a simple attack vector, however, do not have a solution for every possible type of APT attack vector". The conclusion is that "APT vectors can be controlled to some extent, but not 100 percent of the time". Technical users have proposed multiple technical countermeasures to combat APT attacks, that have been segregated into eighteen technical measures. Table 1 illustrates the proposed countermeasures, and their application to the four APT planes. 
Table 1. Technical measure at the four APT planes

\begin{tabular}{|r|l|l|}
\hline \multicolumn{1}{|l|}{ Countermeasures } & \\
\hline 1 & SIEM \\
\hline 2 & Manage security tools \\
\hline 3 & Data loss prevention & \\
\hline 4 & Update \\
\hline 5 & Alerts for privilege escalation \\
\hline 6 & Email filtering \\
\hline 7 & Al anti-spams \\
\hline 8 & IDPS \\
\hline 9 & Email audit \\
\hline 10 & End-point security \\
\hline 11 & Network isolation & \\
\hline 12 & Penetration testing \\
\hline 13 & IP address confidentiality & \\
\hline 14 & Web filters \\
\hline 15 & DMZ (segmenting network) \\
\hline 16 & Threat management gateway & \\
\hline 17 & Security baseline - hardware \\
\hline 18 & \\
\hline
\end{tabular}

In Figure 1 countermeasures are classified into four deduced categories affecting the four planes. From the findings these can be identified as (1) detection systems (alerts, Intrusion Detection Prevention System [IDPS], SIEM, and end point security), (2) email security (email audit, email filtering, AI anti spams), (3) network configuration (network isolation, DMZ, threat management gateway, IP confidentiality, web filtering), and (4) technical management duties (configuring updates, managing security tools, penetration testing, assessing and developing a configuration baseline of hardware, ensuring automated change management, and implementing a strategy for data loss management [DLP]).

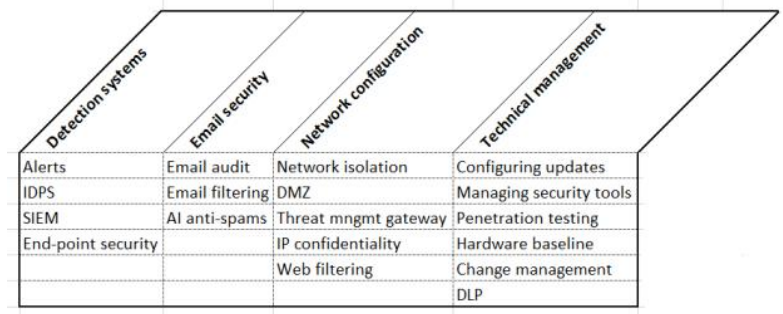

Figure 1. Technical countermeasures for APT

Eighteen technical security measures have been proposed as best practices by the respondents. Installing and configuring of alert rules and mechanisms need to be deployed on all critical devices and databases, where deviance from the normal baseline, is a relevant control. This should be applied at both the client and server level. IDS/IPS rules should be configured with updated signatures to prevent server-based, as well as end-point level attacks. A security incident and event management (SIEM) system should be used to monitor servers, operating systems and applications, to detect abnormal behavior (differing from clearly defined threshold levels). While this has been stated as an effective method, respondents noted that thresholds must be evaluated and applied at all layers. Using End-point security at the user level, USB use as well as unintentional user induced mistakes such as link redirection (to malicious web forms), and malicious downloads needs to be controlled. Respondents stated the "first line of defense is your desktop control".

Regarding email, two technical aspects were proposed. First, an email software audit should be performed to show any abnormal changes performed at the administration level. Second, analysis should be performed to correlate incoming emails and corresponding outgoing email. Respondents emphasized the role of email filtering, due to its effectiveness in filtering spam email and removing malicious attachments, as well as attachments with particular file extensions. Anti-malware solutions in particular systems using artificial intelligence, were proposed as another effective method. This type of solution studies the network/system behavior and adjusts itself to check abnormal communication or non-standard IP packet configuration.

Respondents suggested segmenting or isolating networks as an effective way to prevent the lateral movement of the APT malware, and also prevent privilege escalation. A DMZ with well-defined boundaries should be employed to minimize APT coverage within the organization. A threat management gateway (TMG) should be installed at the boundary to ensure only encrypted data can only enter or exit the network when using appropriate authentication methods at all times. Ensuring IP address confidentiality is critical. Respondents 
suggested against publicly revealing company IP addresses, and ensuring adequate security of DNS servers and architecture. Web filters should be used to limit access to malicious sites, as well as limit access to the company Intranet and extranet.

Two perspectives exist regarding updates. First, all applications need to be updated regularly and second, updates must be pushed to all systems on a regular and automated basis. Security tools should be managed to ensure appropriate installation and configuration of information systems, and security tools across all the four planes. In this respect respondents stated that the IT security manager should endeavor to maintain relevant controls, threats and appropriate configurations relating to identified threats. Third party use of 'white box' and 'black box' tools to test applications and networks. In this respect, the organization gives relevant standards to the third party where they conduct penetration testing based on the selected information security standards. A minimum-security baseline (MSB) should be set for every type of network device or server. Once employed this will allow devices to feed into appropriate applications like SIEM. Since most of the APT attacks occur due to vulnerabilities, automated information technology service management (ITSM) software assists in ensuring approved management policies are adhered to. Finally data loss prevention (DLP) was proposed as an effective method to ensure that end users do not send sensitive or critical information outside the internal network. However, respondents stated that resistance from users often exists, due a perceived loss of flexibility when implemented.

\section{CONCLUSION}

APT style attacks have proved effective at penetrating organizational networks due to their use of 'zero day' malware, advanced penetration techniques, social engineering and stealth. In this research we considered the life cycle of APT attacks as they traverse through four dimensional planes (user, network, application and physical plane). Using interviews with IS security experts, we identified technical control measures that fall into four categories namely detection systems, email security, network configuration and technical management through the four dimensions resultin in eighteen technical measures. Subsequently, the result of this research also presents a set of best practice technical measures that can be applied by organizations for the detection and prevention of APT attacks. In this paper, we have only focused on the technical controls to combat APT attacks where end point security, updates, alerts, email filtering and IDS/IPS (alerts) were highlighted as critical control methods when attempting to counter APT attacks. In addition, SIEM and managing security tools were also found to be useful to ensure protection at all planes.

\section{REFERENCES}

Alshamrani, Adel, Sowmya Myneni, Ankur Chowdhary, and Dijiang Huang. 2019. 'A Survey on Advanced Persistent Threats: Techniques, Solutions, Challenges, and Research Opportunities', IEEE Communications Surveys \& Tutorials.

Aparicio-Navarro, Franciso J, Konstantinos G Kyriakopoulos, Ibrahim Ghafir, Sangarapillai Lambotharan, and Jonathon A Chambers. 2018. "Multi-Stage Attack Detection Using Contextual Information." In MILCOM 2018-2018 IEEE Military Communications Conference (MILCOM), 1-9. IEEE.

Chandra, J Vijaya, Narasimham Challa, and Sai Kiran Pasupuleti. 2016. "Advanced Persistent Threat defense system using self-destructive mechanism for Cloud Security." In 2016 IEEE International Conference on Engineering and Technology (ICETECH), 7-11. IEEE.

Chandran, Saranya, P Hrudya, and Prabaharan Poornachandran. 2015. "An efficient classification model for detecting advanced persistent threat." In Advances in Computing, Communications and Informatics (ICACCI), 2015 International Conference on, 2001-09. IEEE.

Chen, Ping, Lieven Desmet, and Christophe Huygens. 2014. "A Study on Advanced Persistent Threats." In Communications and Multimedia Security, 63-72. Springer.

De Vries, Jelle, Hans Hoogstraaten, Jan van den Berg, and Semir Daskapan. 2012. "Systems for detecting advanced persistent threats: a development roadmap using intelligent data analysis." In Cyber Security (CyberSecurity), 2012 International Conference on, 54-61. IEEE. 
Ghafir, Ibrahim, Vaclav Prenosil, Mohammad Hammoudeh, Francisco J Aparicio-Navarro, Khaled Rabie, and Ahmad Jabban. 2018. "Disguised executable files in spear-phishing emails: Detecting the point of entry in advanced persistent threat." In Proceedings of the 2nd International Conference on Future Networks and Distributed Systems, 44. ACM.

Ghafir, Ibrahim, Vaclav Prenosil, Mohammad Hammoudeh, Liangxiu Han, and Umar Raza. 2017. "Malicious ssl certificate detection: A step towards advanced persistent threat defence." In Proceedings of the International Conference on Future Networks and Distributed Systems, 27. ACM.

Giura, Paul, and Wei Wang. 2013. 'Using Large Scale Distributed Computing to Unveil Advanced Persistent Threats', SCIENCE, 1: pp. 93-105.

Giura, Paul, and Wei Wang. 2012. "A context-based detection framework for advanced persistent threats." In Cyber Security (CyberSecurity), 2012 International Conference on, 69-74. IEEE.

2013. 'Using Large Scale Distributed Computing to Unveil Advanced Persistent Threats', SCIENCE, 1: pp. 93-105.

Huang, Linan, and Quanyan Zhu. 2019. 'Adaptive strategic cyber defense for advanced persistent threats in critical infrastructure networks', ACM SIGMETRICS Performance Evaluation Review, 46: 52-56.

Iwata, Kazuki, Yoshitaka Nakamura, Hiroshi Inamura, and Osamu Takahashi. 2017. "An automatic training system against Advanced Persistent Threat." In 2017 Tenth International Conference on Mobile Computing and Ubiquitous Network (ICMU), 1-2. IEEE.

Jeon, Kyung-Sik, Se-Jeong Park, Sam-Hyun Chun, and Jong-Bae Kim. 2016. 'A Study on the Big Data Log Analysis for Security', International Journal of Security and Its Applications, 10: 13-20.

Marchetti, Mirco, Fabio Pierazzi, Alessandro Guido, and Michele Colajanni. 2016. "Countering Advanced Persistent Threats through security intelligence and big data analytics." In Cyber Conflict (CyCon), 2016 8th International Conference on, 243-61. NATO CCD COE.

Matsuda, Wataru, Mariko Fujimoto, and Takuho Mitsunaga. 2018. "Detecting APT Attacks Against Active Directory Using Machine Leaning." In 2018 IEEE Conference on Application, Information and Network Security (AINS), 60-65. IEEE.

Moon, Daesung, Hyungjin Im, Ikkyun Kim, and Jong Hyuk Park. 2015. 'DTB-IDS: an intrusion detection system based on decision tree using behavior analysis for preventing APT attacks', The Journal of Supercomputing: 1-15.

Moon, Daesung, Hyungjin Im, Jae Dong Lee, and Jong Hyuk Park. 2014. 'MLDS: multi-layer defense system for preventing advanced persistent threats', Symmetry, 6: 997-1010.

Saud, Zainab, and M Hasan Islam. 2015. "Towards proactive detection of advanced persistent threat (apt) attacks using honeypots." In Proceedings of the 8th International Conference on Security of Information and Networks, 154-57. ACM.

Siddiqui, Sana, Muhammad Salman Khan, Ken Ferens, and Witold Kinsner. 2016. "Detecting advanced persistent threats using fractal dimension based machine learning classification." In Proceedings of the 2016 ACM on international workshop on security and privacy analytics, 64-69. ACM.

Thakar, Bhavik, and Chandresh Parekh. 2016. "Advance persistent threat: botnet." In Proceedings of the Second International Conference on Information and Communication Technology for Competitive Strategies, 143. ACM.

Vert, Gregory, Bilal Gonen, and Jayson Brown. 2014. 'A Theoretical Model for Detection of Advanced Persistent Threat in Networks and Systems Using a Finite Angular State Velocity Machine (FAST-VM)', International Journal of Computer Science and Application.

Whittaker, S. 2006. 'Qualitative Researchin Transfusion Medicine: Closing the Gap ', ISBT Science Series, 1: 133 - 39.

Yang, Lu-Xing, Pengdeng Li, Yushu Zhang, Xiaofan Yang, Yong Xiang, and Wanlei Zhou. 2018. 'Effective repair strategy against advanced persistent threat: A differential game approach', IEEE Transactions on Information Forensics and Security.

Zhang, Qingyun, Huan Li, and Jinsong Hu. 2017. "A study on security framework against advanced persistent threat." In 2017 7th IEEE International Conference on Electronics Information and Emergency Communication (ICEIEC), 128-31. IEEE. 